\title{
Stability of a BEC with Higher-order Interactions near a Feshbach Resonance
}

\author{
N.T. Zinner ${ }^{1}$ and M. Thøgersen ${ }^{2}$ \\ 1 Department of Physics, Harvard University, Cambridge, Massachusetts 02138, USA \\ ${ }^{2}$ Department of Physics and Astronomy, University of Aarhus, DK-8000 Aarhus, Denmark
}

(Dated: November 1, 2018)

\begin{abstract}
We consider the stability of an ultracold trapped Bose-Einstein condensate near a Feshbach resonance. Using a modified Gross-Pitaevskii equation that includes higher-order terms and a multichannel model of Feshbach resonances, we find regions where stability can be enhanced or suppressed around experimentally measured resonances. We suggest a number of ways to probe the stability diagram. Using scattering length zero-crossings huge deviations are founds for the critical particle number. Effects are enhanced for narrow resonances or tighter traps. Macroscopic tunneling of the condensate is another possible probe for higher-order interactions, however, to see this requires very narrow resonances or very small particle numbers.
\end{abstract}

PACS numbers: 03.75.Hh,03.75.Lm,67.85.-d,67.85.Bc

Introduction The stability of Bose-Einstein condensates (BECs) in ultracold alkali gases is determined by the sign of the scattering length $a$ [1]. For $a<0$, one has effectively attractive interactions and the condensate will collapse to a dense state when the number of condensed particles $N$ is larger than a critical number $N_{c}$ $[1,2,3,4]$. This has been beautifully demonstrated in experiments with ${ }^{7} \mathrm{Li}$ 5], ${ }^{87} \mathrm{Rb}$ 6, 7], and recently with a dipolar ${ }^{52} \mathrm{Cr} \mathrm{BEC}[8]$. The findings indicate that the theory based on the surprisingly simple Gross-Pitaevskii (GP) equation can reproduce and describe most features of the experiments.

The GP equation includes two-body terms through a contact interaction which is parametrized by $a$. This is equivalent to a Born approximation but with an effective coupling that is obtained by replacing $a_{b o r n}$ by the physical scattering length $a$. However, higher-order terms in the expansion of the phase shifts at low momenta, determined by the effective range $r_{e}$, the shape parameter etc., give corrections to the simple GP equation. In this paper we explore the influence of the effective range term on the properties of a BEC. In particular, we show that the critical number of condensed atoms depends strongly on the higher-order scattering term when the scattering length approaches zero (zero-crossing). We also show how the macroscopic quantum tunneling (MQT) rate, in which the entire BEC tunnels as a coherent entity, can be modified for small condensate samples.

The considered effects depend on a combination of $a$ and $r_{e}$ which yield different behavior for wide and narrow Feshbach resonances. Recent measurements on ${ }^{39} \mathrm{~K}$ found many both wide and narrow resonances which allow for tuning of $a$ over many orders of magnitude [9, 10]. We therefore consider a selected example from ${ }^{39} \mathrm{~K}$ in order to elucidate the general behavior for realistic experimental parameters.

The paper is organized as follows: We first introduce the modified GP equation with effective range dependence. Using both a variational and numerical approach we find a phase diagram describing the stability of the BEC. We then consider a Feshbach resonance model including effective range variations. The behavior of the critical particle number near a scattering length zerocrossing is derived. We discuss MQT and show numerically how the rate is modified. We finally discuss other possibilities for probing the higher-order interactions.

Modified GP Equation We assume that the condensate can be described by the GP equation and we focus on the $a<0$ attractively interacting case. Since we are interested in the ultracold regime, where the temperature is much smaller than the critical temperature for condensation, we adopt the $T=0$ formalism. In order to include higher-order effects in the two-body scattering dynamics, we use the modified GP equation derived in 11] for which the equivalent energy functional is

$$
\begin{aligned}
E(\Psi)=\int \mathrm{d} \boldsymbol{r} & {\left[\frac{\hbar^{2}}{2 m}|\nabla \Psi|^{2}+V(\boldsymbol{r})|\Psi|^{2}\right.} \\
+ & \left.\frac{U_{0}}{2}\left(|\Psi|^{4}+g_{2}|\Psi|^{2} \nabla^{2}|\Psi|^{2}\right)\right],
\end{aligned}
$$

where $m$ is the atomic mass, $V$ is the external trap, $U_{0}=$ $4 \pi \hbar^{2} a / m$, and $g_{2}=a^{2} / 3-a r_{e} / 2$ with $a$ and $r_{e}$ being the $s$-wave scattering length and effective range, respectively.

We are interested in the stability properties of the ground-state and we therefore perform a variational calculation using the mean-field trial wavefunction

$$
\Psi(r)=\frac{\sqrt{N}}{\pi^{3 / 4} \sqrt{(q b)^{3}}} \exp \left(-\frac{r^{2}}{(q b)^{2}}\right),
$$

where $q$ is the dimensionless variational parameter and $b=\sqrt{\hbar / m \omega}$ is the trap length. The normalization is $N=\int \mathrm{d} \boldsymbol{r}|\Psi(r)|^{2}$. For simplicity we only consider isotropic traps with $V(r)=\frac{1}{2} m \omega^{2} r^{2}$. However, the effects found should hold for deformed traps as well (along the lines of the analysis in [12]). The variational energy is

$$
\frac{E(q)}{N \hbar \omega}=\frac{3}{4} q^{2}+\frac{3}{4} \frac{1}{q^{2}}+\frac{1}{\sqrt{2 \pi}} \frac{N|a|}{b}\left(-\frac{1}{q^{3}}+3 \frac{g_{2}}{b^{2}} \frac{1}{q^{5}}\right) .
$$


In Fig. 1 we plot $E(q)$ for different parameters. As shown, there are many possibilities for $g_{2} \neq 0$, including stable, unstable, and metastable systems. We see that the $g_{2}$ term modifies the barrier for $N|a| / b=0.5$, implying that tunneling rates will be altered. For $N|a| / b=0.7$, the $g_{2}=0$ case has no barrier at all, and here the $g_{2}$ term can in fact produce a small barrier on its own. The $q^{-5}$ dependence of the term means that the effect is small. However, the plot clearly shows that a new stability analysis is needed. In addition to the variational approach we have numerically solved the full time-independent GP equation corresponding to eq. (11).
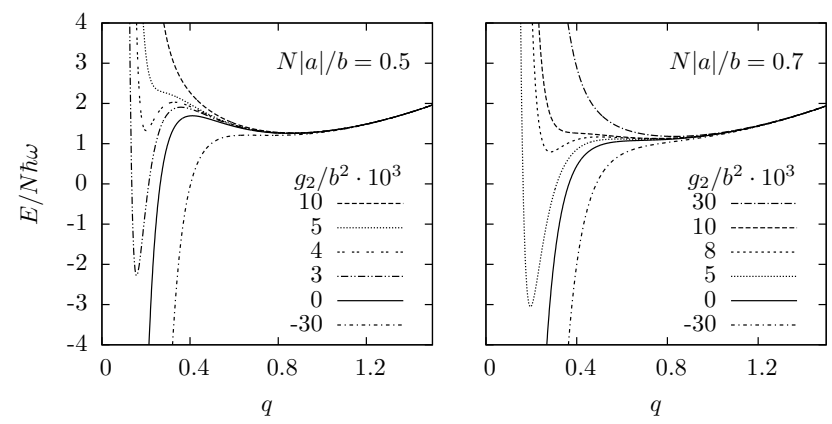

FIG. 1: Energy of a BEC with fixed $N|a| / b$ as function of the variational parameter $q$, i.e. the size of the condensate. The higher-order interaction term $g_{2}$ modifies the height and shape of the barrier.

Phase Stability Diagram To determine the groundstate stability one looks for the vanishing of the barrier towards $q=0$. For $g_{2}=0$ eq. (3) leads to $N_{c}|a| / b \approx 0.671[1]$. The full integration of the GP equation gives $N_{c}|a| / b \approx k_{0}, k_{0}=0.5746$ [2, 3]. These values are indicated by filled points in Fig. 2.

In the general $g_{2} \neq 0$ case we first take the variational energy eq. (3) and solve for multiple roots of $\mathrm{d} E(q) / \mathrm{d} q$. The resulting "phase diagram" is plotted in Fig. 2 (solid line). In the upper left region (S) we have complete stability of the condensate; only one minimum exists at large $q \sim 0.8$ (see Fig. 11) and the potential goes to plus infinity at small $q$. In the metastable region (M) a barrier and a minimum exists for large $q$; for $g_{2}>0$ another minimum exists at small $q \lesssim 0.2$, while for $g_{2}<0$ the potential goes to minus infinity. In the unstable region $(\mathrm{U})$ the barrier has vanished; the potential either has a minimum at small $q\left(g_{2}>0\right)$ or no minimum at all $\left(g_{2}<0\right)$. In the variational approach the stable and unstable regions are connected via the upper right part of Fig. 2, Going from $(\mathrm{S})$ to $(\mathrm{U})$ corresponds to an adiabatic change, where the macroscopic $(q \sim 1)$ minimum is transferred to a microscopic $(q \ll 1)$ high-density minimum.

Next, we numerically solve the full the GP equation. The stationary (white) and non-stationary (shaded) regions are shown in Fig. 2. Since both stable and metastable solutions are considered stationary, the white regions cover both $(\mathrm{S})$ and $(\mathrm{M})$. Both our variational and

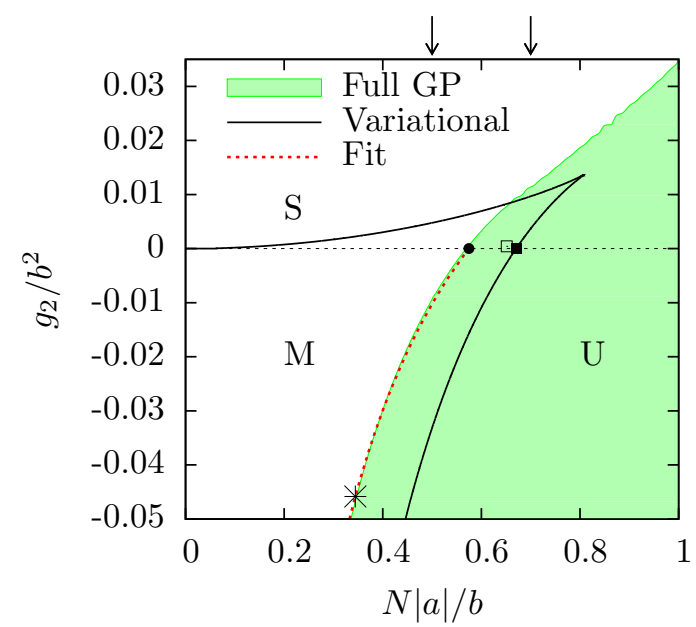

FIG. 2: (color online) Phase stability diagram of a BEC with higher-order interactions. The solid line obtained from the variational ansatz, eq. (2), divides the stable (S), metastable $(\mathrm{M})$, and unstable $(\mathrm{U})$ regions. The white region indicates where stationary solutions exists for the full GP equation. Filled points show the well-known $g_{2}=0$ results. Arrows correspond to the values in Fig. 1. The dashed line is the fit in eq. (5). Two specific ${ }^{39} \mathrm{~K}$ values are chosen for zero-crossing (cross) and MQT rates (open square) calculations (see text).

numerical results agrees with the known $g_{2}=0$ results, and have similar behavior for small and negative $g_{2} / b^{2}$.

Feshbach Resonance Model In order to predict effects of the higher-order term, we need a realistic model for $g_{2}$. Since $g_{2} / b^{2}$ is the relevant parameter, and the trap length, $b$, is usually orders of magnitude larger than atomic scales, it is necessary to look for divergences of $g_{2}$. Since $g_{2}$ depends on $a$ and $r_{e}$, a Feshbach resonance is the obvious mean. The standard single-channel models are inadequate since only $a$ is considered. We therefore use a multi-channel model [13], which describes both $a$ and $r_{e}$ as a function of resonance position $B_{0}$, width $\Delta B$, magnetic moment difference between the channels $\Delta \mu$, and the background scattering length $a_{b g}$. In [13], only the regime $\left|B-B_{0}\right| \ll \Delta B$ was of interest, yielding $r_{e}$ independent of magnetic field, $B$. In the current work we generalize to keep the $B$-dependence of both $a$ and $r_{e}$. This gives $a=a_{b g}\left(1-\Delta B /\left(B-B_{0}\right)\right)$ and $r_{e}=r_{e 0} /(1-$ $\left.\left(B-B_{0}\right) / \Delta B\right)^{2}$, where $r_{e 0}=-2 \hbar^{2} / m a_{b g} \Delta \mu \Delta B<0$. We thus have a field-dependent $g_{2}(B)$ for given values of $B_{0}$, $\Delta B, \Delta \mu$, and $a_{b g}$. Notice that $r_{e}=r_{e 0}\left(1-a_{b g} / a\right)^{2}$ and

$$
g_{2}(a)=\frac{a^{2}}{3}-\frac{a r_{e 0}}{2}\left(1-\frac{a_{b g}}{a}\right)^{2} .
$$

Hence $g_{2}$ diverges when $a \rightarrow 0$ (referred to as zerocrossing) or $a \rightarrow \infty$ (on resonance). These are wellknown features for model potentials like the square-well and van der Waals interactions.

As a concrete example we use the extremely narrow Feshbach resonance in ${ }^{39} \mathrm{~K}$ found at $B_{0}=825 \mathrm{G}$, with 


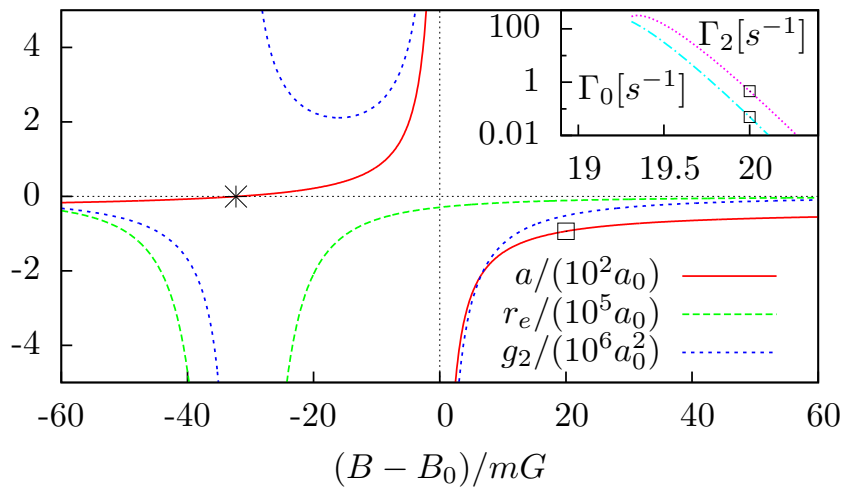

FIG. 3: (color online) Scattering length $a$, effective range $r_{e}$, and coupling constant $g_{2}$ as function of $B$-field for the narrow ${ }^{39} \mathrm{~K}$ Feshbach resonance at $B_{0}=825 \mathrm{G}$. The inset shows the MQT rate $\Gamma_{2}$ (and $\Gamma_{0}$ for $g_{2}=0$ ) for $N=242$ and $b=1.84 \mu \mathrm{m}$. The cross and open square are as in Fig. 2

$\Delta B=-0.032 \mathrm{G}, \Delta \mu=3.92 \mu_{B}$, and $a_{b g}=-36 a_{0}[9]$. For this resonance we find a large $r_{e 0}=-2.93 \times 10^{4} a_{0}$. The variation of $a, r_{e}$ and $g_{2}$ as function of $B$ is shown in Fig. 3. We use trap length $b=1.84 \mu \mathrm{m}=3.48 \times 10^{4} a_{0}$ in all calculations unless indicated otherwise.

Critical Particle Number Near Zero-crossings As noted above, large values of $g_{2}$ are possible at zerocrossings $(a=0)$ since $r_{e}$ diverges here, see e.g. Fig. 3 around $B-B_{0} \sim-32 \mathrm{mG}$. The scattering length is currently being tuned with extreme accuracy near such zerocrossings in ${ }^{39} \mathrm{~K}$ around $350 \mathrm{G}$ with control down to the $0.06 a_{0}$ level [14]. However, this is for a broad resonance and the same level of tuning around narrow ones is likely much more challenging, particularly in high magnetic fields. Another narrow resonance at $B_{0}=25.85 \mathrm{G}$ with $\Delta B=0.47 \mathrm{G}$ exists in ${ }^{39} \mathrm{~K}[9]$. It will show the same features as the one considered here and the lower magnetic field could make tuning easier.

The advantage is that many particles $N_{c} \propto 1 /|a|$ can be accommodated in the condensate. However, for $a \rightarrow 0$ we have $a g_{2} \rightarrow-r_{e 0}\left(a_{b g}\right)^{2} / 2$, i.e. a finite limit. Remembering that $r_{e 0}$ and $\nabla^{2}|\Psi|^{2}$ are negative, the last term in the energy functional eq. (1) also becomes negative. Thus larger densities or density fluctuations gives lower total energy. This implies less stability and smaller $N_{c}$ near $a=0$.

To calculate quantitative effects on $N_{c}$ we focus on the critical line between the unstable and metastable regions in Fig. 2 for $g_{2} \leq 0$. We fit the dependency as

$$
N_{c}=k_{0} \frac{b}{|a|} \times\left(1-k_{1} \frac{g_{2}(a)}{b^{2}}\right)^{-1}
$$

where $k_{1}=14.5$, as shown in Fig. 2, With $g_{2}(a)$ given by eq. (4), $N_{c}$ becomes a function of $|a| / b$ for fixed values of $a_{b g} / b$ and $r_{e 0} / b$. In Fig. 4 we plot $1 / N_{c}$ as function of $|a| / b$ for the ${ }^{39} \mathrm{~K}$ resonance with various trap lengths. The curve deviates from the linear $g_{2}=0$ result both

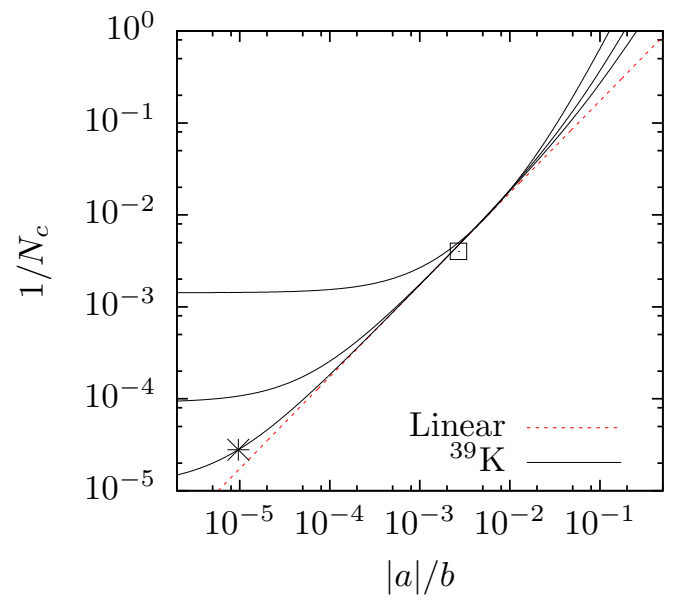

FIG. 4: (color online) Critical particle number $N_{c}$ as function of scattering length $a$. The linear dashed (red) line is the well-known $g_{2}=0$ result $N|a| / b=k_{0}$. The solid (black) lines are for a narrow ${ }^{39} \mathrm{~K}$ resonance and trap length $b=1.84 \mu \mathrm{m}$, (bottom), $0.5 b$ (middle), and $0.2 b$ (top). The cross and open square are as in Fig. 2

at $|a| \sim 0$ and $|a| \sim b$. Squeezing the trap makes the effect even more pronounced. In the limit $|a| / b \rightarrow 0$ the critical particle number approaches a finite value, $N_{c} \rightarrow$ $2 k_{0} b^{3} /\left(k_{1}\left|r_{e 0}\right| a_{b g}^{2}\right)$. Large effects also occur near $|a| \sim b$, however, here the allowed particle number is very small.

To exemplify with the ${ }^{39} \mathrm{~K}$ resonance we use $B-B_{0}=$ $-32.3 \mathrm{mG}$ (cross in Fig. 3) giving $a=-0.334 a_{0}$ and $g_{2} / b^{2}=-0.0461$. The critical particle number from eq. (5) is $N_{c}=3.58 \times 10^{4}$. The values are indicated by crosses in Fig. 2 and Fig. 4 . The $g_{2}=0$ prediction is $N_{c}=5.975 \times 10^{4}$. In conclusion, we predict $40 \%$ reduction in $N_{c}$ for macroscopic particle number $N_{c} \sim 10^{4}$.

Macroscopic Quantum Tunneling Close to $a=\infty$, $g_{2} / b^{2}$ is still a small term in typical traps. As the MQT rate [15] is exponentially dependent on the integral under the barrier, we expect changes due to non-zero $g_{2}$ to be amplified. A similar point was raised in [12] where the MQT rate was shown to increase dramatically close to $N_{c}$. The MQT rate, $\Gamma_{2}$, can be obtained from field theory [17] by considering the bounce solution of the effective action in variational $q$-space with potential $E(q) / N \hbar \omega$. All MQT calculations are done within the variational approach, eq. (3). Since we assume spherical symmetry, we use $\omega=\bar{\omega}=\left(\omega_{x} \omega_{y} \omega_{z}\right)^{1 / 3}$ when relating to experiment. For comparison, we denote by $\Gamma_{0}$ the rate with $g_{2}=0$.

We are interested in metastable states with small barriers and large MQT rates, thus we have to work close to $N_{c}$ [12]. Below we find that $g_{2} / b^{2}$ is only important for the rate when $N$ is of order $10^{2}$ or less. Since the threebody loss depends on the density to the third power [16], we expect it to be small in the outer metastable minimum and to be large in the inner one. Thus, the physical picture is that of a metastable BEC in the outer minimum 
that knocks on the barrier as a coherent state with a common quantum tunneling probability. When it tunnels to the inner minimum it swiftly decays as the large increase in density amplifies the three-body loss. Thermal fluctuations can of course also induce MQT and we therefore need to operate at very low temperature. An estimate of the thermal tunneling rate is given in 17], and leads to the following criterion for thermal fluctuations to be suppressed: $E\left(q_{m}\right)-E\left(q_{0}\right) \gg k_{B} T$, where $q_{m}$ and $q_{0}$ are the positions of the barrier maximum and the outer minimum, respectively.

For the ${ }^{39} \mathrm{~K}$ resonance we now consider $B-B_{0}=20 \mathrm{mG}$ (see Fig. 3) where $g_{2} / b^{2}=-4.3 \times 10^{-4}$ and $a=-93.6 a_{0}$. The critical particle number becomes $N_{c} \simeq 249$ (see Fig. (4). We now pick $N=242<N_{c}$ to obtain metastability (see Fig. 2). The rates are found to be $\Gamma_{2}=0.49$ $\mathrm{s}^{-1}$ and $\Gamma_{0}=0.05 \mathrm{~s}^{-1}$ (shown by the open squares in the inset of Fig. 3), i.e. a tenfold enhancement of the MQT rate. The temperature must be below $8 \mathrm{nK}$ for thermal fluctuations to be small. It is possible to obtain larger $N$ with the cost of a smaller effect on the MQT rate. However, going to $N \sim 10^{3}$ does not seem possible.

We propose to start from the $a>0$ side where the condensate is stable. Preparing a sample with low enough $N$ and temperature of a few $\mathrm{nK}$ for the effect to be observable is the very difficult challenge for the MQT scenario. A sudden ramp of the magnetic field to the appropriate $a<0$ is then performed. The system has to retain a small barrier so that the MQT rate is considerable and to avoid collapse. Then one monitors $N$ as a function of time, as was done in e.g. [7]. The low $N$ is also challenging for optical imaging of the atomic cloud, but should be possible with current techniques. We expect not only to see modified MQT rates, but also changes in the decay after the condensate tunnels to a high density state; The three-body recombination is very sensitive to the density, and the inner barrier caused by the $g_{2}$ term should affect this process as well.

Other Signatures From the structure of the $g_{2}$ term it is clear that density variations are needed to see effects. As we have demonstrated already, tighter traps amplify the contribution, and we expect optical lattices to do the same. Solitons and vortices are other features with density variation. For a simple one vortex state, the $|\Psi|^{2} \nabla^{2}|\Psi|^{2}$ term will leave the core and the asymptotic regions unchanged but change the profile in between. Work is in progress to extract these changes [18].

Conclusion We have explored the effect of higherorder terms in the Gross-Pitaevskii description of a BoseEinstein condensate, particularly the effective range correction near a Feshbach resonance. Using both a variational and numerical approach we find an interesting new phase diagram for the stability of a condensate with negative scattering length.

The critical particle number is strongly affected near zero-crossings of the scattering length for narrow reso- nances. Effects of $40 \%$ are found for particle number of order $10^{4}$. These deviations increase for more narrow resonances or tighter traps. We find that the critical number will be reduced as the zero-crossing is approached from either side since the higher-order term is always attractive. The dipole-dipole interaction is usually important near zero-crossings but we estimate it to be smaller than the higher-order term for narrow resonances and tight traps. Macroscopic quantum tunneling is also modified by these higher-order corrections and we have discussed some experimental conditions for exploring the physics. Narrow Feshbach resonances are the best way to isolate the effect of tunneling. However, one needs small samples of order $10^{2}$ particles and temperatures around $10 \mathrm{nK}$. Other possible experimental signatures are tight traps, optical lattices, solitons, and vortices in rotating BECs.

In a more general sense, the fact that higher-order interactions can become large and dominant in determining the stability properties means that it is no longer a correction and that still higher terms might become important. This is the subject of future work. For the moment we have demonstrated that experiments targeting the regions discussed above could find interesting new stability properties beyond those that were already understood about a decade ago.

Acknowledgments Discussions and encouragement from Aksel S. Jensen is gratefully appreciated. NTZ acknowledges useful comments from Doron Gazit, as well as support from the Villum Kann Rasmussen foundation.

[1] F. Dalfovo, S. Giorgini, L.P. Pitaevskii, and S. Stringari, Rev. Mod. Phys. 71, 463 (1999).

[2] P.A. Ruprecht, M.J. Holland, K. Burnett, and M. Edwards, Phys. Rev. A 51, 4704 (1995).

[3] A. Gammal, T. Frederico, and L. Tomio, Phys. Rev. A 64, 055602 (2001).

[4] G. Baym and C.J. Pethick, Phys. Rev. Lett. 76, 6 (1996).

[5] C.C. Bradley, C.A. Sackett, and R.G. Hulet, Phys. Rev. Lett. 78, 985 (1997).

[6] J. L. Roberts et al., Phys. Rev. Lett. 86, 4211 (2001).

[7] E.A. Donley, Nature 412, 295 (2001).

[8] T. Lahaye et al., Phys. Rev. Lett. 101, 080401 (2008).

[9] C. D'Errico et al., New J. Phys. 9, 223 (2007).

[10] G. Roati et al., Phys. Rev. Lett. 99, 010403 (2007).

[11] A. Collin, P. Massignan, and C.J. Pethick, Phys. Rev. A 75, 013615 (2007).

[12] M. Ueda and A.J. Leggett, Phys. Rev. Lett. 80, 1576 (1998).

[13] G.M. Bruun, A.D. Jackson, and E.E. Kolomeitsev, Phys. Rev. A 71, 052713 (2005).

[14] M. Fattori et al., Phys. Rev. Lett. 101, 190405 (2008).

[15] A nice introduction to MQT with references is found in D. Mozyrsky et al., Phys. Rev. A 76, 051601(R) (2007).

[16] S.K. Adhikari, Phys. Rev. A 66, 013611 (2002).

[17] H.T.C. Stoof, J. Stat. Phys. 87, 1353 (1997).

[18] M. Thøgersen and N.T. Zinner, in preparation. 